Voix et Images

voixetimages

\title{
V.6 BJ/NBJ : difficile modernité
}

\section{Pierre Nepveu}

Volume 10, numéro 2, hiver 1985

La barre du jour / La nouvelle barre du jour

URI : https://id.erudit.org/iderudit/013881ar

DOI : https://doi.org/10.7202/013881ar

Aller au sommaire du numéro

Éditeur(s)

Université du Québec à Montréal

ISSN

0318-9201 (imprimé)

1705-933X (numérique)

Découvrir la revue

Citer cet article

Nepveu, P. (1985). V.6 BJ/NBJ : difficile modernité. Voix et Images, 10(2),

159-165. https://doi.org/10.7202/013881ar

Ce document est protégé par la loi sur le droit d'auteur. L'utilisation des services d'Érudit (y compris la reproduction) est assujettie à sa politique d'utilisation que vous pouvez consulter en ligne.

https://apropos.erudit.org/fr/usagers/politique-dutilisation/ 


\title{
V.6 BJ/NBJ: difficile modernité
}

\author{
par Pierre Nepveu, Université de Montréal
}

Qu'est-ce que la modernité? La "nouvelle écriture» est-elle autre chose qu'un désir de changement (pour le changement)? Qu'est-ce qu'être moderne? La NBJ a consacré, on le sait, ses deux colloques «bissextiles» (29 février 1980 et 1984) à ces questions, sans toutefois aborder des questions plus profondes, les seules en définitive qui puissent donner sens aux premières: pourquoi un groupe d'écrivains québécois des années quatrevingt sent-il le besoin de se réclamer de la modernité, après Baudelaire, Rimbaud et toutes les avant-gardes d'une bonne partie du vingtième siècle? Que signifie la revendication du «moderne» maintenant, c'est-à-dire historiquement? Si une écriture est «moderne» lorsqu'elle est branchée sur la réalité contemporaine, lorsqu'elle est dynamique et critique, qu'elle transforme et questionne, alors que reste-t-il de valeur opératoire, de simple pertinence da un concept si dilué qu'il finit par désigner tout ce qui n'est pas académique et rétrograde? Et si la «nouvelle écriture» ne vit, comme l'observait justement Hugues Corriveau en 1980, «que de sa propre absence de définition», pourquoi cet acharnement à définir?

Commençons donc par la fin, meme si cette fin est relative et que la NBJ continue. La sur-utilisation du terme de «modernité», le ressassement exaspéré auquel il donne lieu, les contestations (pour la première fois venues de l'intérieur même du mouvement, et non d'un anti-modernisme primaire) qu'il suscite, tout cela indique assez que nous touchons depuis 1980 un point-limite. Si la modernité est, comme l'a déjà écrit Octavio Paz, une «tradition de la rupture», elle doit logiquement et nécessairement en venir à se nier elle-même, et elle ne peut que mourir de trop s'expliciter, ou de se figer dans une sorte d'incantation magique.

Des définitions, des formules, il n'en manque pourtant pas. En voici, jalonnant le colloque de 1984 (NBJ, no 141), une liste non exhaustive: la modernité, c'est «la dialectique parole/acte, texte/écrivant, privé/politique, écriture/lecture»; c'est «l'hétérogène, la destruction des fétiches, la langue éventrée, une démarche individuée, une épreuve du sens, le goat du réel, les femmes-en-sont-plus-proches-que-les-hommes»; c'est «l'anonymat, le procès de la signifiance, le refus de la symbolique (des pères), le TEXTE comme genre littéraire, une figure du temps, le gout du mouvement et de la transformation». Est-ce assez? Cela sans parler des définitions négatives, contestant l'appellation: la modernité serait devenue «une bannière, une marque, un copyright, une négation de l'écriture, une norme, un refuge dans le groupe, une peur de la singularité».

Le problème n'est donc pas que l'on soit à court de définitions, mais que somme toute, ces définitions soient si courtes, je veux dire si peu 
compréhensives et si dépourvues de perspective historique. Imprégnée dès son origine par une volonté de mise à mort de la littérature et de l'histoire, la BJ est toujours restée marquée par ces négations qui continuent de la hanter de diverses manières. Ainsi France Théoret, au colloque de 1980, en venait à se demander si la nouvelle écriture ne serait pas «née du refoulement de la critique et de la théorie» (NBJ, no 90-91). Etonnant paradoxe pour un mouvement qui s'est voulu plus critique et théorique qu'aucun autre dans toute l'histoire de la littérature québécoise. Un paradoxe que Théoret éclaire en expliquant qu'il y aurait eu deux façons de concevoir la modernité à la BJ, l'une valorisant la pratique, l'autre insistant sur l'illisible. Tendance pragmatique: la pratique serait en elle-même une critique, la fiction véhiculerait sa propre théorie. Tendance nihiliste: la théorie risque de réintroduire le sens, la littérature, et de transformer en capital symbolique une illisibilité subversive. Dans les deux cas, critique et théorie n'ont plus guère de place. La littérature veut se définir comme un acte (d'écriture), la modernité consiste à produire moins une ouvre qu'un événement, comme voulaient le faire Hubert Aquin dans Prochain épisode et Paul Chamberland dans l'Afficheur hurle. En substituant le mot "écriture» au mot «littérature», la pratique moderniste préconisée à la BJ cherchait à être performative, un agir plutôt qu'un dire. Le rêve rimbaldien refait surface: «la poésie ne rythmera plus l'action, elle sera en avant», c'est-à-dire ellemême action et révolution.

Dès 1968, Claude Bertrand définit le «texte», qui sera la notion et l'arme-clés de cette conception: «Nous employons ici le mot «texte» dans son acception sémiologique, c'est-à-dire qu'il doit être considéré comme une pratique. Cette pratique, ou texte, est un tout fermé, ordonné, cohérent, justifié (BJ, no 14, 1968). Et dans le numéro suivant, consacré au jeune poète disparu Jean-Michel Valiquette, Bertrand et Marcel St-Pierre présentent celui-ci comme un "poète de la négation absolue et de l'illisible» contestant «le texte de la société québécoise» (BJ, no 15, 1968). Texte moderne versus texte social, là se joue l'événement. Mais nous n'en sommes pas à un paradoxe près. D'abord, voici une «pratique» qui se définit par une fermeture: mieux encore, c'est la clôture du texte qui en garantit l'efficacité. Il y a davantage: en contestant la «pseudo-rationalité») de la société bourgeoise, le texte moderne propose une rationalité fort étrange, qui consiste à construire un système ordonné et justifié de négations folles, incontrôlables, une organisation cohérente de l'incohérence, une sémiologie du non-sens. Aquin déjà avait parlé d'une «forme informe».

En ce sens, la BJ proposait à la société et au milieu littéraire des années soixante un message à double contrainte: «vous devez nous lire - vous ne pourrez pas nous lire». Mais cette double contrainte, la revue elle-même doit la vivre, et il est significatif de voir Marcel St-Pierre réclamer en 1969-1970, dans un document interne, que l'on accorde autant de place a la critique et à la réflexion théorique qu'à la création puisque, «la BJ, dit-il, vise à l'élaboration claire d'une stratégie littéraire susceptible de déborder (c'est moi qui souligne) sur les plans culturel, social et politique». 
Ce vou, on le sait à présent, ne sera jamais proche de se réaliser. Ce n'est pas tant que l'illisible, tautologiquement, ne peut être lu et pensé, car aucun texte ne saurait être fait intégralement de négations pures. C'est plutôt que le texte moderne, version $B J$, ne peut conserver sa force transgressive et négatrice qu'en régissant à l'avance son lecteur, quel qu'il soit. "Nouvelle écriture» implique d'entrée de jeu «nouvelle lecture», et celle-ci passe par une auto-lecture (du texte par lui-mème). Contre la «fraude» et le "gros bon sens littéraire», contre la lecture rêveuse nourrissant les profondeurs de la subjectivité romantico-bourgeoise, contre la récupération idéologique, Nicole Brossard réclamera une lecture sans ailleurs, ici-maintenant, "précise» et «en surface». Voild où la fermeture du texte prend sons sens: un certain illisible, apte en principe à faire délirer le lecteur, y devient plutôt le principe d'une lisibilité normative. En ce sens, la "nouvelle écriture» est une écriture de pouvoir: le texte ne déroute son lecteur que pour mieux le maîtriser, pour le forcer à «bien lire», et il se lit lui-même de peur qu'on ne le lise «ailleurs» ou «autrement«. Rien d'étonnant, dès lors, à ce que le statut de la critique et de la théorie soit si problématique: celles-ci courent toujours le risque d'être superfétatoires, ou pire, de réintroduire une lecture déracinante, voire idéologique. Dilemme fondamental de la modernité depuis Baudelaire, comme le montre Octavio Paz dans le Rire et la Pénitence: l'absolue négation, dans le texte moderne, est aussi une utopie de la présence absolue, de l'absolument présent, mais cela signifie en fait une présence désincarnée, une présence du vide et au vide. L'hyper-rationalisme de la modernité débouche, suprême ironie, sur une religion.

Ce que l'on a appelé le "fétichisme du texte» coinncide, dans l'histoire de la BJ, avec l'apogée du structuralisme dans les milieux d'enseignement québécois, apogée à laquelle la revue fait écho en 1973 avec son numéro intitulé Onze analyses (BJ, no 39-41). Ces lectures rigoureusement intratextuelles, mettant à jour la cohérence et l'auto-suffisance du texte, illustrent une des conséquences possibles de la théorie du texte moderne à la BJ. La mise à mort de l'histoire et de la littérature est aussi mise à mort de la lecture, et ces meurtres rituels débouchent non pas sur la révolution, mais sur le technocratisme, autant efficace qu'amnésique, miroir de la société libérale de la post-Révolution tranquille.

Mais il y a une autre conséquénce possible: c'est le type de lecture qui se développera à la NBJ, durant la phase d'institutionnalisation de la modernité québécoise. Lecture-miroir, lecture qui mime son objet dans' un incessant rituel de célébration et parvient par là seul à échapper à la pure redondance. La modernité critique devient ici une modernité euphorique et romantique, applaudissant les «textes-voltiges» et la «star écriture» (Claude Beausoleil), et privilégiant la "connivence» et la "complicité», comme en témoigne par exemple le colloque consacré à Nicole Brossard en 1982 (NBJ, no 118-119). Entre la normativité et la célébration, l'espace reste étroit pour une modernité compréhensive s'interrogeant sur son propre contenu et son rapport au réel. Ce que l'affrontement de 1984 entre la NBJ et les Herbes 
rouges montre assez, en faisant éclater en pleine lumière la contradiction entre rationalisme et irrationalisme, jamais suffisamment pensée à la $\mathrm{BJ}$; et plus encore, en posant la question du réel, que celui-ci soit défini comme «politique», «féministe», "expérience du monde», "subjectivité radicale», «Enfer» ou «Paradis». Question du réel, c'est-à-dire: question du contenu.

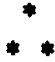

C'est seulement avec la NBJ, après 1977, que l'usage du terme «modernité») s'est vraiment réparidu, au moment où sous l'impact du mouvement féministe notamment, la pratique moderne du texte paraît forcée de se redéfinir d'une manière moins négative, ce qui donne lieu à une «nouvelle lisibilité», expression d'une naïveté déconcertante, tant par l'appauvrissement qu'elle trahit dans la pensée littéraire québécoise des années soixante-dix, que par le danger qu'elle fait courir à la pratique du texte telle que définie à la BJ. Normand de Bellefeuille l'a bien senti qui, après avoir défendu cette notion de lisibilité dans la revue, la critique vigoureusement au colloque de 1980 sur la «Nouvelle écriture». En ce sens, le mot «modernité» vient chapeauter tardivement un mouvement où les tensions et les contradictions deviennent plus virulentes.

L'adjectif «moderne» est lui-même d'un usage beaucoup plus restreint que l'on pourrait croire dans l'histoire de la BJ, entre 1965 et 1977 . Il est fréquemment recouvert a l'origine par des termes comme «nouveauté, recherche (formelle), évolution», et il demeure le plus souvent implicite dans les références à des pratiques québécoises antérieures où la BJ se reconnaît (Giguère, les Automatistes), ou derrière des notions comme «texte» ou «transgression». Mais l'idée du moderne est partout dans l'air au moment de la fondation de la revue. Comme en Europe à l'époque de Marx et de Baudelaire, cette idée est liée à un changement profond de société, à un enthousiasme qui croit tous les progrès permis et possibles. Un des mots-clés de la Révolution tranquille est modernisation, ce qui signifie, 'chez les politiciens, économistes et idéologues (Pierre Trudeau et René Lévesque sont, à l'époque, d'accord là-dessus): démocratie, société pluraliste, croissance économique, rationalité bureaucratique et étatique, et sur le plan psychologique: confiance en soi, lucidité, critique de tous les mythes. Et c'est sous le signe d'un «dynamisme nouveau» et d'une «inévitable situation de lucidité» que la BJ se présentera dès son premier numéro: le groupe fondateur, en février 1965, croit encore qu'il est possible d'écrire "dans le sens de l'histoire», que progrès social et progrès littéraire vont de pair.

Si la poésie du pays, à laquelle s'opposera la BJ, a eu un tel impact entre 1960 et 1965 , c'est notamment qu'elle a paru donner un contenu prophétique, eschatologique et mythique à cette modernisation de la société québécoise. Pourtant, le malentendu éclate dans la mesure où cette poésie dénonce aussi la modernisation, en commençant à percevoir la réalité sur le mode du manque (thèmes de l'aliénation, de l'exil, de la dépossession, etc.). 
L'idée du texte moderne prend racine là où cette négativité du vécu social (négativité liée en partie seulement au problème national) n'est plus assumée sur le mode nostalgique (nostalgie de l'unité, de la totalité retrouvées) mais, comme chez Aquin et Chamberland, sur un mode actif et performatif, par le moyen d'une esthétique elle-mème négative, voulant saboter la (belle) littérature de peur de cautionner l'invivable.

C'est Chamberland qui effectue le plus directement le relais (et la coupure) entre la poésie du pays et la BJ. Rien n'est plus éloquent que l'extrait de l'Inavouable que la BJ publie en 1966 (no 6), où Chamberland peut encore écrire, dans un élan de lyrisme amoureux, qu'il «vibre au centre du monde», alors qu'une page plus tôt, il a parlé d'un «monde dur, hostile, étranger». La conscience urbaine, l'un des traits dominants de la modernité à la BJ, se dépolitise et s'irréalise pour déboucher sur le couple conscience malheureuse / fascination. La ville est invivable et fascinante, mais dans tous les cas, elle est étrange, exotique. La modernisation nous a conduits dans les limbes.

«Le signe de la modernité est un stigmate: la présence blessée par le temps; tatouée par la mort», écrit Octavio Paz; «la modernité habite un non-lieu", affirme pour sa part Shoshana Felman. Curieux renversement: l'optimisme progressiste de la Révolution tranquille accouche d'un terrible (mais envoatant) vertige. On ne finirait plus de citer des textes de la BJ, surtout d'avant 1970 , qui témoignent de ce vertige. Etre moderne, c'est alors regarder «lucidement» le trou, c'est-à-dire refuser de le combler par quelque contenu (mythe, idéologie, nostalgie) que ce soit. Valiquette: «tout m'anéantit (...) un abîme impossible sera mon nom» (BJ, no 8, 1966); Théoret: «Partir de rien/ rien n'est/ néant/ pur refus/ pure absence» (ibid.). Il ne s'agit surtout pas d'une expérience tragique, car le tragique est cela même qui cautionne selon Bròssard la littérature. C'est une expérience intellectuelle, une pratique raisonnée du vacuum, en même temps qu'une arme contre le lecteur, comme on peut encore le voir en 1974: "Cette écriture ne pardonne pas qui enchaîne ici votre regard et qui ne vous avance à rien sinon au bord de l'abîme que vous craignez comme un trou" (BJ, no 44).

Dans un texte qui a l'allure d'un manifeste a postoriori, paru en 1970, Nicole Brossard et Roger Soublière écrivaient: «nous avons intellectualisé notre mal» (BJ, no 26). L'aveu est de taille: le tabou de cette modernité, c'est la souffrance (d'où l'hédonisme du texte qui deviendra un thème des années soixante-dix). L'intellectualisation consiste à faire le vide plutôt qu'à le subir, et l'envoutement qui en découle est celui de la transgression, que Brossard distingue de la simple agression. La transgression (à laquelle la revue consacre son numéro 42 , en 1973), c'est précisément l'expérience (non tragique) de l'abîme, expérience vécue comme un éternel préambule, comme la promesse d'une connaissance à venir, comme l'espérance de contenus encore informulables. 
Il est significatif que, dans le numéro sur la transgression, la seule tentative pour faire déboucher celle-ci sur un contenu précis et positif soit celle de François Charron, mais qu'elle relève du petit catéchisme marxistemaoïste, qui constituera au milieu des années soixante-dix une matière étonnamment irréaliste de reposer la question du réel, et une négation complète de la modernité. La transgression ne peut rester moderne qu'en côtoyant le vide, en «multipliant les actes marginaux jusqu'à ce qu'ils aient un sens» (Brossard, BJ, no 29, 1971). Un sens différé, non idéologique, dont tout ce qu'on peut dire, c'est qu'il sera nouveau, transformateur.

Dans le paysage littéraire des années soixante-dix, il ne serait pas exagéré de dire que le féminisme a représenté pour le projet moderniste une planche de salut, en procurant un contenu historique tangible à l'entreprise de déconstruction. La NBJ, il faut le rappeler, a été lancée en 1977 à partir d'un constat relativement morose et comme un projet de relance: «...donner à lire des textes qui puissent à nouveau rendre effervescente et stimulante une littérature qui, pour le moment, paraît hésitante» (NBJ, no 58).

Au colloque consacré en 1982 à Nicole Brossard, Louise Dupré devait parler du féminisme comme d'un «détournement de la modernité» (NBJ, no 118-119). L'expression indique assez que la relation entre féminisme et modernité n'est pas simple et qu'elle peut même dans certains cas être contradctoire. Une des conséquences les plus importantes de la montée de l'écriture des femmes a été de lever le tabou sur la souffrance et par là, de remettre en cause l'intellectualisme du projet moderne. Plus encore, alors qu'on parlait à la fin des années soixante d'un ceffondrement du symbolique», on commence à évoquer un «retour du symbolique», comme le fait déja Madeleine Gagnon dans le Corps, les mots, l'imaginaire, numéro spécial (BJ, no 56-57, 1977) qui précède immédiatement l'avènement de la NBJ. Et ce simple titre: «La mère peut-elle être moderne?» (NBJ, no 116, 1982), montre du moins que la chose ne va pas de soi, même si la réponse est somme toute positive, comme chez Monique La Rue: "La mère moderne dit: je suis un corps sexué qui parle». Même chez Nicole Brossard, le thème de la clôture du texte se nuance: «L'écriture, quand on n'en sort pas, et je ne pense pas en sortir, est son commencement et sa fin. Mais il lui faut une obsession de taille pour l'accompagner, obsession qui se nourrit sans doute d'un qui-suis-je dans la langue qui me parle intérieurement; $j$ 'entends ici non pas la langue maternelle, mais une langue figurative, tellement figurative que pour vivre avec, je dois en abstraire l'essentiel. Pour Joyce, l'Irlande; pour moi, la femme» (NBJ, no 118-119, 1982). Comment mieux dire que la Femme est devenue le contenu ou, par un imprévu retour des choses: le Pays de la modernité.

Une chose est sare: en même temps que les mots «moderne» et «modernité» deviennent avec la NBJ sur-utilisés, leur contenu paraît de plus 
en plus flou, contradictoire. Le mot «moderne» devient davantage un mot magique, un pathos aiguillonnant le sujet-écrivant qu'une catégorie critique et opératoire. Que le mot ait toujours eu, depuis son utilisation par Baudelaire et surtout Rimbaud, cette valeur rhétorique et stratégique, c'est certain, mais ces auteurs se situaient à une époque où la notion d'art moderne commençait à peine à se formuler, comme critique de la représentation et de toutes les valeurs humanistes instaurées par la Renaissance. Plus d'un siècle plus tard, et à un moment où le modernisme est remis en question dans le domaine des arts visuels, cette rhétorique fait problème et le moins qu'on puisse dire, c'est qu'elle appelle une critique vigoureuse que le débat NBJ/Herbes rouges de 1984 amorce peut-être.

En particulier, l'opposition écriture/littérature, allant jusqu'à la négation frivole et irresponsable du phénomène littéraire (et à un moment où la «modernité» est devenue précisément un mouvement littéraire, institutionnalisé) ne peut plus équivaloir qu'à une démission de la pensée. Par ailleurs, comme tout mouvement idéologique, le féminisme a aussi produit ses interdits, ses stéréotypes, ses écritures parasitaires. Qu'il soit devenu souvent le contenu principal du projet «moderne» à la NBJ implique du même coup que la modernité risque de devenir une simple enveloppe, une série de critères ou de formes réifiées. Lorsque Jean Yves Collette écrit à un auteur refusé que la NBJ privilégie les textes de la «marginalité formelle», il y aurait lieu de se demander justement de quelle marge il s'agit, et si la définition peut n'être que formelle.

Dans une perspective historique plus large, la revendication du moderne par des écrivains québécois contemporains ne peut que manifester un doute profond sur la place et la fonction sociale de l'écrivain. Il faut rappeler que la BJ est apparue à une époque où, comme l'ont écrit Brossard et Soublière en 1970 (BJ, no 26), «sur le terrain poético-politique, tout semblait avoir été dit». La modernité est l'activation paradoxale d'un désoeuvrement, elle y puise son énergie mais elle y éprouve en même temps son impuissance, qui est aussi celle de l'ensemble de la classe intellectuelle et écrivante, comme en témoigne amèrement le numéro de la NBJ (130-131, 1983) consacré à l'Intellectuel/le en 1984. Dès lors, on peut s'étonner à bon droit, avec François Charron (les Herbes rouges, no 123-124), que la modernité se réclame tout à coup du Réel, mais il reste que le réel est le grand Autre de la subjectivité moderne, désiré nostalgiquement, nommé comme impossible, aussi exotique que séduisant, aussi impensable qu'incontournable. 